\title{
The Structure of Baroclinic Modes in the Presence of Baroclinic Mean Flow
}

\author{
K. H. BRINK AND J. PEDLOSKY \\ Woods Hole Oceanographic Institution, Woods Hole, Massachusetts
}

(Manuscript received 17 May 2019, in final form 5 November 2019)

\begin{abstract}
This contribution seeks to understand the vertical structure of linearized quasigeostrophic baroclinic modes when they are modified by the presence of a baroclinic mean flow and associated potential vorticity gradients. It is found that even modest, $O\left(0.05 \mathrm{~m} \mathrm{~s}^{-1}\right)$, mean flows can give rise to very substantial changes in modal structures, often in the sense of increased surface intensification. The extent to which stable modes are modified depends strongly on the direction of Rossby wave propagation. Further, baroclinically unstable solutions can appear, and a meaningful inviscid critical-layer solution can occur at the transition to instability when the horizontal gradient of potential vorticity changes sign at some depth within the water column. In addition, the gravest, $n=0$, vertical stable mode is no longer strictly barotropic, but rather it can carry density variability at frequencies much higher than those possible for baroclinic (higher) Rossby wave modes. This finding appears to be consistent with oceanic current-meter observations that suggest temperature variability propagation even when the frequency is too high for traditional baroclinic Rossby waves to exist.
\end{abstract}

\section{Introduction}

In recent years, accumulating records of ocean currents have shown that subinertial current fluctuations in the open ocean have a vertical distribution inconsistent with that of a single, simple linear flat-bottom baroclinic mode (Wunsch 1997; Sanchez de la Lama et al. 2016). Specifically, in many (but not all) cases, currents at depth are weaker than might be expected based on an $n=1$ vertical mode with realistic density profiles, and (in these cases) there is generally no current reversal with depth. This result can be expressed either as a phase locking of the barotropic and first baroclinic modes, or in terms of modes that are required to vanish at depth. Indeed, Wortham and Wunsch (2014) found that modified, bottom pressure decoupling (BPD) modes, which have no horizontal flow at the bottom, are a better representation of oceanic variability than are the conventional modes. Thus, there has been recent interest in effects that might modify the traditional stable baroclinic modes [see Wortham and Wunsch (2014) for further references]. For example, Samelson (1992) and Bobrovich and Reznik (1999) show that bottom corrugations can lead to weakened near-bottom Rossby wave velocities. LaCasce (2017) argues that bottom slopes on a larger spatial scale often lead to modified baroclinic

Corresponding author: K. H. Brink, kbrink@whoi.edu modes effectively having a bottom boundary condition of zero horizontal velocity rather than zero vertical velocity (but results depend strongly on propagation direction). Also, it can be shown that bottom friction can lead to near-zero horizontal velocities near the bottom, although with realistic parameters and scales, this mechanism appears unlikely in the deep ocean (Brink and Pedlosky 2018).

We seek to explore one other mechanism by which the traditional stable baroclinic modes can be strongly modified (beyond the well-known surface intensification associated with a realistic density profile that has stronger stratification near the surface). Specifically, Charney and Drazin (1961) showed that vertically propagating disturbances in the atmosphere can be strongly modified by an ambient potential vorticity gradient. In practice, vertically sheared mean currents are associated through geostrophy with horizontal density gradients and these variations, in turn, represent a modification of the ambient potential vorticity field. Since standing vertical modes result from the reflection of vertically propagating signals, it seems clear that modifications in the oceanic potential vorticity field will lead to changes in the vertical normal modes in the ocean. The question then is how strong this modification might be under realistic conditions? One example that touches on this question is that of Killworth et al. (1997), who addressed the extent to 
which oceanic mean flows affect the westward propagation speed of baroclinic Rossby waves. Their study, however, is restricted to strictly zonal mean flow, long Rossby waves and east-west propagation. Under these conditions, they were able to address issues of zonal propagation speed, but they were not motivated to explore the extent to which modal structures can be changed via ambient potential vorticity. Another relevant study is that of Smith (2007) who focuses on the properties of baroclinically unstable modes, but does not consider the structure of the stable modes.

Here, we seek to examine in more detail how ambient potential vorticity fields affect the structure of baroclinic modes, particularly at the energy-containing (mesoscale) scales in the midlatitude ocean. In particular, we consider linearized quasigeostrophic waves in a continuously stratified, flat-bottom ocean, using stratification and velocity estimates derived from ocean climatology. The directions of both the mean flow and of wave propagation are unconstrained in our approach. We thus deal with both baroclinic instabilities and with criticallayer phenomena, although we focus on vertical structure rather than instability per se. Further, we consider how these results may help explain an observed tendency for temperature information to propagate westward in the ocean at a frequency too high for traditional baroclinic Rossby waves.

\section{Analysis}

The quasigeostrophic vorticity equation, linearized about a mean horizontal flow $[\overline{u(z)}, \overline{v(z)}]$, can be written as

$$
q_{t}+\bar{u} q_{x}+\bar{v} q_{y}+u \Pi_{x}+v \Pi_{y}=0
$$

(e.g., Pedlosky 1992, chapter 7), where the perturbation quasigeostrophic potential vorticity (QGPV) is

$$
q=v_{x}-u_{y}-g f \rho_{0}^{-1}\left(\frac{\rho}{N^{2}}\right)_{z}
$$

and the mean QGPV (with horizontally uniform mean flows) is

$$
\Pi=f+\beta y-g f \rho_{0}^{-1}\left(\frac{\bar{\rho}}{N^{2}}\right)_{z} .
$$

The Coriolis parameter is given by $f$, the northward ( $y$ ) gradient of $f$ is given by $\beta$, the buoyancy frequency is $N$, and subscripted independent variables represent partial differentiation. The mean density $\bar{\rho}$ varies in the vertical direction, and it varies linearly in $(x, y)$. Perturbation velocity, density, and pressure are $(u, v), \rho$, and $p$, respectively. Now, using geostrophy to represent $(u, v)$ and assuming that

$$
p=P(z) \exp [i(k x+l y-\omega t)],
$$

(1a) becomes

$$
0=\omega^{\prime}\left[-\left(k^{2}+l^{2}\right) P+f^{2}\left(\frac{P_{z}}{N^{2}}\right)_{z}\right]+\left(-k \Pi_{y}+l \Pi_{x}\right) P,
$$

where

$$
\omega^{\prime}=\omega-k \bar{u}-l \bar{v}
$$

and the potential vorticity gradient is given by

$$
\begin{aligned}
& \Pi_{y}=\beta-f^{2}\left(\frac{\bar{u}_{z}}{N^{2}}\right)_{z} \\
& \Pi_{x}=f^{2}\left(\frac{\bar{v}_{z}}{N^{2}}\right)_{z} .
\end{aligned}
$$

Barotropic instabilities are not possible because the mean flow is horizontally uniform in our midocean approximation. Assuming a flat bottom and a rigid lid, the boundary conditions (derived directly from the equation governing density changes) are

$$
\omega^{\prime} P_{z}=\omega_{z}^{\prime} P \quad \text { at } \quad z=-D, 0,
$$

where $D$ is the constant ocean depth. For comparison, we point out that in a resting, flat-bottom ocean both the surface and bottom boundary conditions are $P_{z}=0$, whereas the BPD assumption would make the bottom boundary condition simply $P=0$. Some of the more unusual notation is summarized in Table 1.

It is straightforward [following, e.g., Pedlosky (1992, section 7.4)] to derive the necessary condition for instability:

$$
\begin{aligned}
0= & f^{2}\left[\left(|P|^{2} \frac{k \bar{u}_{z}+l \bar{v}_{z}}{N^{2}\left|\omega^{\prime}\right|^{2}}\right)_{z=0}-\left(|P|^{2} \frac{k \bar{u}_{z}+l \bar{v}_{z}}{N^{2}\left|\omega^{\prime}\right|^{2}}\right)_{z=-D}\right] \\
& +\int_{-D}^{0} \frac{|P|^{2}}{\left|\omega^{\prime}\right|^{2}}\left(k \Pi_{y}-l \Pi_{x}\right) d z .
\end{aligned}
$$

The application of this result differs from that in, say, Charney's (1947) atmospheric case because in the ocean the first (free surface) term in brackets dominates the second (because of weak flow at the bottom); in the atmosphere, the second (Earth's surface) term is expected to dominate. Thus, for zonal flow with constant shear, surface-intensified westward oceanic surface flow $\left(\overline{u_{z}}<0\right)$ 
TABLE 1. Some mathematical symbols.

\begin{tabular}{cl}
\hline \hline Symbol & \multicolumn{1}{c}{ Definition } \\
\hline$c$ & Scalar phase speed in the critical-layer problem $=\omega / K$ \\
$K$ & Scalar wavenumber $[(5 \mathrm{~b})]$ \\
$Q$ & Gradient of mean field potential vorticity $\Pi[$ see $(11 \mathrm{~b})]$ \\
$s$ & Inverse depth scale in fits for mean velocity or \\
& buoyancy frequency \\
$\bar{U}$ & Mean flow in the direction of the wavenumber [(11a)] \\
$\gamma$ & Ratio of surface modal amplitude to bottom amplitude \\
$\eta$ & Ratio of max (as a function of $z)$ modal amplitude \\
& to min amplitude \\
$\Pi$ & Mean field potential vorticity $[(1 \mathrm{c})]$ \\
$\omega^{\prime}$ & Doppler-shifted wave frequency $[(3 \mathrm{~b})]$ \\
\hline
\end{tabular}

will be potentially unstable because of the contrast between the surface term and the integrand (assuming that $\Pi_{y}$ has the same sign as $\beta$ ), but in the atmosphere it is eastward flow $\left(\overline{u_{z}}>0\right)$ that would satisfy this condition strongly.

Further, the results from Pedlosky (1992, section 7.5) are generalized to show that, for unstable waves, the real part of the frequency is bounded by

$$
\min (k \bar{u}+l \bar{v})-\frac{\beta k}{2 K^{2}}<\omega_{R}<\max (k \bar{u}+l \bar{v}),
$$

where the scalar wavenumber is

$$
K^{2}=k^{2}+l^{2}
$$

and the scalar wavelength $\lambda=2 \pi / K$. Critical layers (where $\omega^{\prime}=0$ ) are possible over the range (shaded in Figs. 2, 5, 6, 8, and 9, below)

$$
\min (k \bar{u}+l \bar{v})<\omega<\max (k \bar{u}+l \bar{v}) .
$$

The problem (3) is solved by a two-step numerical process. First, the solution is expanded in terms of the inviscid, linear, flat-bottom baroclinic modes in the absence of a mean flow. These obey

$$
0=\frac{1}{c_{n}^{2}} F_{n}+\left(\frac{F_{n z}}{N^{2}}\right)_{z}
$$

subject to

$$
F_{n z}=0 \quad \text { at } \quad z=-D, 0 .
$$

By taking advantage of the orthogonality condition

$$
\delta_{n m}=\int_{-D}^{0} F_{n} F_{m} d z
$$

the solution to (3) can be found as an algebraic eigenvalue problem for $\omega$ and $a_{n}$,

$$
0=\sum_{m} B_{n m} a_{m}-\omega a_{n}
$$

where

$$
\begin{aligned}
P= & \sum_{m} a_{m} F_{m}, \\
B_{n m}= & -\left(b_{n m}+d_{n m}+g_{n m}\right)\left(k^{2}+l^{2}+\frac{f^{2}}{c_{n}^{2}}\right)^{-1}, \\
b_{n m}= & f^{2}\left\{\left[\left(k \bar{u}_{z}+l \bar{v}_{z}\right) \frac{F_{n} F_{m}}{N^{2}}\right]_{z=0}\right. \\
& \left.-\left[\left(k \bar{u}_{z}+l \bar{v}_{z}\right) \frac{F_{n} F_{m}}{N^{2}}\right]_{z=-D}\right\}, \\
d_{n m}= & f^{2} \int_{-D}^{0}\left(k \bar{u}_{z}+l \bar{v}_{z}\right) \frac{1}{N^{2}}\left(F_{m} F_{n z}-F_{n} F_{m z}\right) d z, \\
g_{n m}= & \int_{-D}^{0}\left[-(k \bar{u}+l \bar{v})\left(k^{2}+l^{2}+\frac{f^{2}}{c_{n}^{2}}\right)\right. \\
& \left.+k \Pi_{y}-l \Pi_{x}\right] F_{n} F_{m} d z .
\end{aligned}
$$

The coefficients (7c)-(7f) are found numerically for a given $(k, l)$. This problem is then solved using usually 190 eigenfunctions $F_{n}$ to obtain estimates for $\omega$, although 390 were sometimes used when more accuracy was required. Many of the solutions resulting from this calculation are rejected because $\omega^{\prime}$ passes through zero at some depth. While these numerical solutions are not actually singular, they would become so as the number of eigenfunctions $F_{n}$ becomes large. The finite-difference-grid version of this issue would be a numerical solution where $\omega^{\prime}$ passes through zero, but where the actual zero does not fall on a grid point. As the grid becomes finer, the zero becomes closer to a grid point, thus making the numerical solution more nearly singular. We thus reject inviscid solutions where $\omega^{\prime}$ passes through zero at a depth where the mean vorticity advection is nonzero as nonphysical: the numerical expression of a critical-layer singularity.

More refined solutions for $\omega$ are then found by means of resonance iteration on a finite difference grid. This approach allows better vertical resolution (hence better frequency estimates) and further eliminates other potentially singular solutions. Solutions are obtained by providing arbitrary forcings (two types: one smoothly distributed over depth, and one concentrated near the surface) to the left-hand side of (3a), solving on a finite-difference grid, and searching in complex frequency 
to find the resonant response that is defined by a maximum of

$$
\int_{-D}^{0}\left|P^{2}\right| d z
$$

The nonsingular solutions to (7a) are used as initial estimates for this approach. Vertical grid spacing of $0.5-0.7 \mathrm{~m}$ provides good resolution of modal structures that can have vertical scales as short as $10 \mathrm{~m}$. When a resonant frequency is obtained, the structure of the response ought to be the modal structure, and not a reflection of the arbitrary forcing distribution. Using this two-step approach is useful because the first step, the algebraic eigenvalue problem, is not likely to miss any valid solutions, and the finite-difference approach provides good spatial resolution, hence more accurate estimates of $\omega$. Since this approach is entirely numerical, there is always a possibility that physically meaningful solutions will be missed. However, experimentation gives us confidence, at least for $|\omega|>1 \times 10^{-9} \mathrm{~s}^{-1}$, that we are not missing any meaningful solutions. Inviscid stable solutions are numbered $n=0,1,2, \ldots$, where $n$ is the number of zero crossings in $P$ as a function of depth.

The solutions to (7) can contain singularities associated with critical layers where $\omega^{\prime}(z)=0$ at some depth. To deal with these singularities, it is sometimes convenient to include a constant vertical viscosity (but not diffusivity) $\nu$ to the problem so that (3a) becomes

$$
\begin{aligned}
0= & \omega^{\prime}\left[-\left(k^{2}+l^{2}\right) P+f^{2}\left(\frac{P_{z}}{N^{2}}\right)_{z}\right]+\left(-k \Pi_{y}+l \Pi_{x}\right) P \\
& +i \nu\left(k^{2}+l^{2}\right) P_{z z}
\end{aligned}
$$

and the boundary conditions (3e) remain unchanged. The algebraic eigenvalue problem (7) is then modified accordingly. For all calculations shown here, the only nonzero value of $\nu$ that we use is $\nu=1 \times 10^{-4} \mathrm{~m}^{2} \mathrm{~s}^{-1}$. Increasing this value by an order of magnitude usually leads to only small (less than $1 \%$ ) changes in the real part of $\omega$ for the solutions we present. When $\nu \neq 0$, the modified version of the necessary condition for instability (4) becomes too complicated to be of much use. Adding viscosity deals with the potential critical-layer singularity by assuring that the frequency is complex. Because we do not allow vertical diffusion, the approach does not increase the order of (9) and so it does not allow the higher order effects that would appear in a more thorough critical-layer treatment.

For calculations with finite viscosity, no solutions of the algebraic eigenvalue calculation [the frictional form of (7)] are dropped because of the $\omega^{\prime}=0$ criterion, since $\omega^{\prime}$ is always complex and thus cannot pass through zero for realistic mean flows. In this case, the 50-100 solutions to (7) with the largest $|\omega|$ are all run through the resonance iteration searches. There is then a nearreplication of the inviscid results (with a weak viscous modification), plus a small number of new solutions that are essentially critical layers made into physically acceptable results because of the frictional damping.

For better numerical stability, we use functional fits for stratification and mean velocity. The buoyancy frequency squared is taken to have the form

$$
N^{2}=N_{0}^{2} \exp \left(s_{N} z\right)
$$

so that the solutions for $\left(c_{n}, F_{n}\right)$ have a convenient analytical form involving Bessel functions (e.g., Brink and Pedlosky 2018). Further, the velocity profiles are parameterized as, for example,

$$
\begin{aligned}
& \bar{u}(z)=U_{1} \exp \left(s_{u 1} z\right)+U_{2} \exp \left(s_{u 2} z\right) \quad \text { and } \\
& \bar{v}(z)=V_{1} \exp \left(s_{v 1} z\right)+V_{2} \exp \left(s_{v 2} z\right) .
\end{aligned}
$$

Values for $N_{0}^{2}$ and $s_{N}$ are found using a least squares fit to climatological density fields for the North Atlantic Ocean from the World Ocean Atlas 2013 (e.g., Zweng et al. 2013). The horizontal resolution of this database is $1^{\circ}$. Velocity fields are either taken to be idealized forms (with $U_{2}, V_{2}=0$ ) or to be more realistic forms found by fitting (10b) and (10c) to velocities calculated from the World Ocean Atlas 2013 mean density fields and referenced to $2000 \mathrm{~m}$ (Table 3), below which data density usually falls off quickly . Examples of these fits are given in Fig. 1. Although (10a) never provides a very good fit, using a two-exponential fit for buoyancy frequency squared [as in (10b) as opposed to (10a)] generally makes only a modest improvement in the fit but would make the problem more difficult by requiring the $F_{n}$ to be computed numerically. We are satisfied, however, that for the present purposes (10a) at least provides results of the right magnitude.

The general approach toward understanding the importance of the mean potential vorticity field for the modal structure is to pick a location [hence set of parameters for (10a)-(10c)] and a scalar wavelength $\lambda$. We concentrate on the choice $\lambda=200 \mathrm{~km}$, which we take to be representative of the energetic ocean mesoscale. Then, the problem is solved for a range of wavenumber directions $\theta$ (measured counterclockwise relative to the east). We begin, however, by treating some simplified, idealized cases in order to explore the phenomenology systematically. Primary model configurations are summarized in Table 2. 

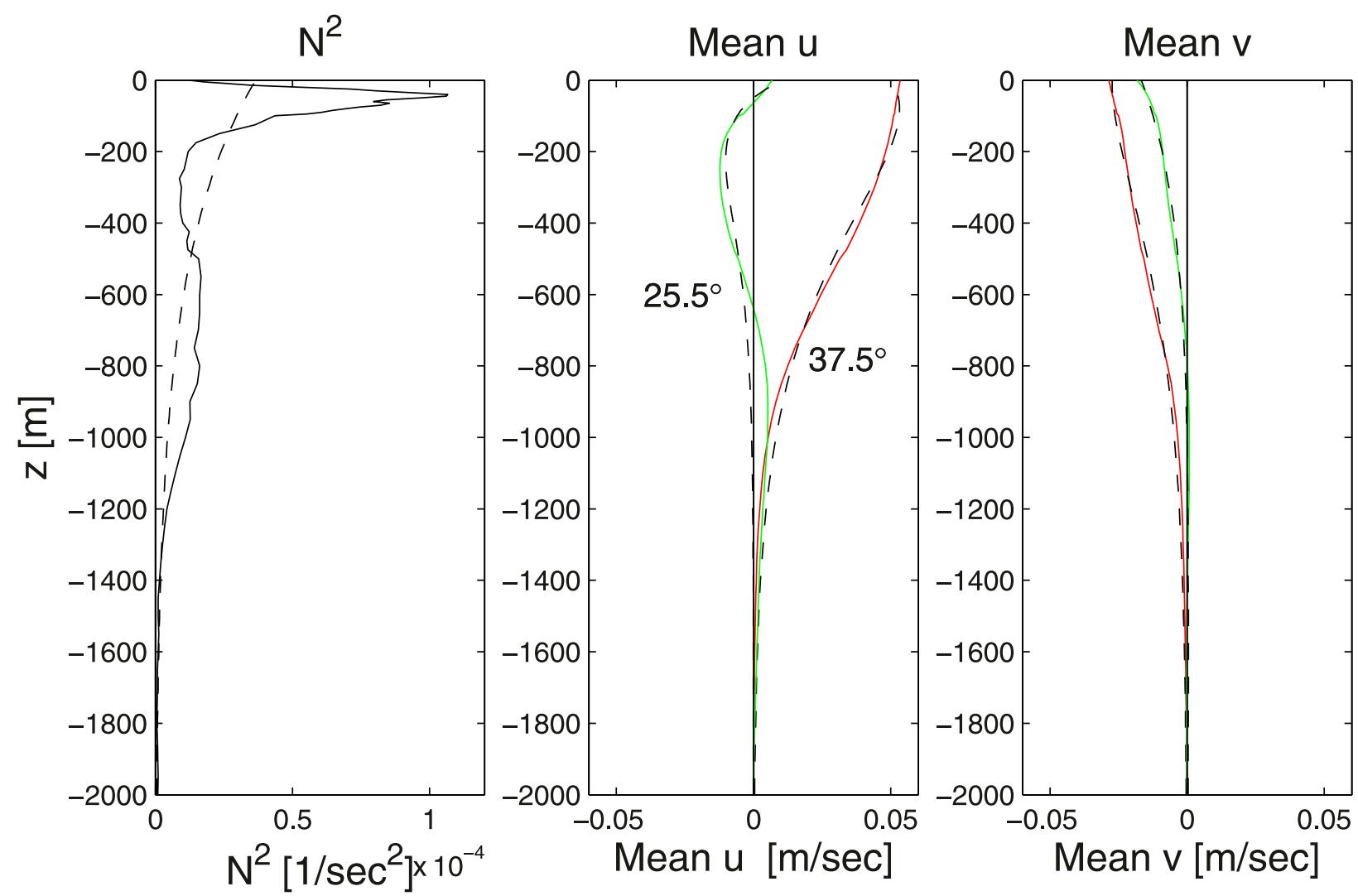

FIG. 1. Examples of climatological stratification [(left) $37.5^{\circ} \mathrm{N}, 50.5^{\circ} \mathrm{W}$, as used in cases $1-4$, and velocity relative to $2000 \mathrm{~m}$ : (center) eastward mean velocity (red line: $37.5^{\circ} \mathrm{N}, 50.5^{\circ} \mathrm{W}$, as in case 4 ; green line: $25.5^{\circ} \mathrm{N}, 50.5^{\circ} \mathrm{W}$ ) and (right) mean northward velocity]. Only the upper $2000 \mathrm{~m}$ are shown. Dashed lines are functional fits (a single exponential for $N^{2}$ and the sum of two exponentials for $u$ and $v$ ) as used in our calculations.

\section{Results}

\section{a. Idealized cases}

\section{1) ZONAL MEAN FLOWS}

Case 1 represents a simple configuration where $N^{2}, f$, and $\beta$ are given by values at $37.5^{\circ} \mathrm{N}, 50.5^{\circ} \mathrm{W}$ (in the Gulf Stream Extension region), but the mean flow is strictly westward $\left(U_{1}=-0.05 \mathrm{~m} \mathrm{~s}^{-1}, U_{2}=V_{1}=V_{2}=0\right.$, and $\left.s_{u 1}=0.0035 \mathrm{~m}^{-1}\right)$. The scalar wavelength is $\lambda=200 \mathrm{~km}$. In this case, the inviscid solution, for all wave orientations $\theta$ (measured counterclockwise relative to east), is simply a complex conjugate pair that includes a baroclinically unstable wave (Fig. 2). The gray-shaded region in Fig. 2 represents the frequency range where critical layers are possible [from (5c)]. For all orientations, the necessary condition for baroclinic instability (4) is met because of the sign difference between the first (surface) term and the integrand. This finding is denoted by the continuous cyan-colored line at the bottom of the figure. There are no solutions for this $\lambda$ with strictly real frequency, that is, stable wave modes. For somewhat larger wavelengths $(\lambda \geq 500 \mathrm{~km})$, a stable $n=0$ mode appears.

TABLE 2. Summary of cases presented.

\begin{tabular}{ccccccc}
\hline \hline Case & Lon $\left({ }^{\circ} \mathrm{W}\right)$ & Lat $\left({ }^{\circ} \mathrm{N}\right)$ & $\lambda(\mathrm{km})$ & $\overline{u_{S}}\left(\mathrm{~m} \mathrm{~s}^{-1}\right)^{\mathrm{a}}$ & $\overline{v_{S}}\left(\mathrm{~m} \mathrm{~s}^{-1}\right)^{\mathrm{a}}$ & Comment \\
\hline 1 & 50.5 & 37.5 & 200 & -0.05 & 0 & Idealized flow \\
2 & 50.5 & 37.5 & 200 & 0.05 & 0.05 & Idealized flow \\
3 & 50.5 & 37.5 & 200 & 0 & -0.027 & Idealized flow \\
4 & 50.5 & 37.5 & 200 & 0.050 & -0.025 & Realistic flow \\
5 & 50.5 & 35.5 & 200 & -0.025 & Realistic flow \\
\hline
\end{tabular}

\footnotetext{
${ }^{\text {a }}$ Surface velocity values.
} 


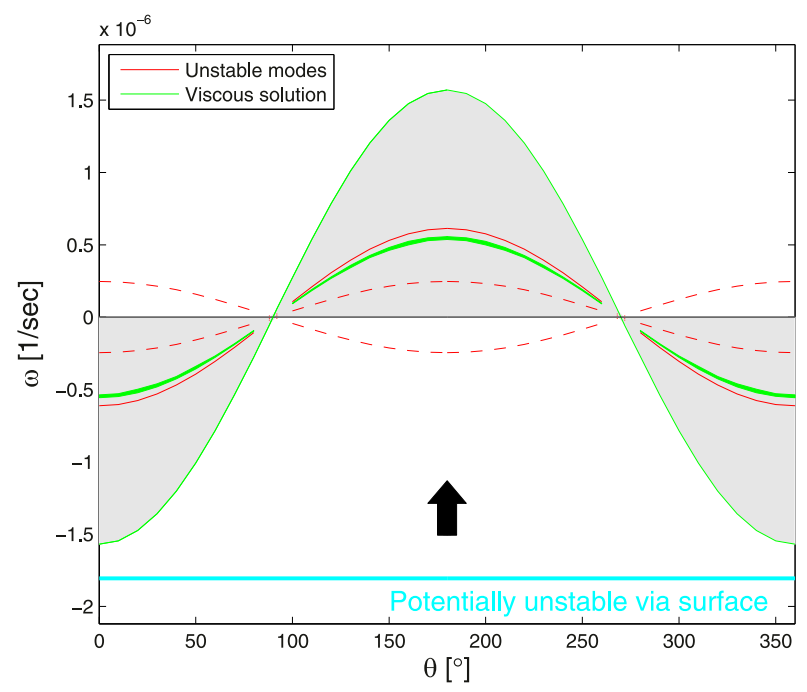

FIG. 2. Frequency of wave solutions vs wave direction for $\lambda=200 \mathrm{~km}$, case $1\left(37.5^{\circ} \mathrm{N}, 50.5^{\circ} \mathrm{W}, U_{1}=-0.05 \mathrm{~m} \mathrm{~s}^{-1}, U_{2}=V_{1}=\right.$ $V_{2}=0$, and $s_{u 1}=0.0035 \mathrm{~m}^{-1}$ ). The solid red line is the real part of frequency for the inviscid complex mode; dashed red lines are the imaginary part of frequency for the same mode, where the positive values represent unstable waves and negative values represent decaying waves; the heavy green line is the real part of frequency for the dense band of solutions when viscosity is allowed (the thickness of the curve spans the range of the dense band); and the lighter green line is the real part of frequency for the surface-trapped viscous solution [for both viscous solutions, the imaginary part is negative and $\left.O\left(10^{-10} \mathrm{~s}^{-1}\right)\right]$. The grayshaded area is the frequency range over which critical layers are possible, given the mean flow and $\theta$. When a viscosity of $\nu=1 \times 10^{-4} \mathrm{~m}^{2} \mathrm{~s}^{-1}$ is included, the red curves are not noticeably changed at this scale. The horizontal cyan line indicates that, for all $\theta$, the system is potentially baroclinically unstable because the first term in (4) differs in sign from the integral. The heavy black arrow indicates the direction of the mean flow. There are no inviscid critical layers for this case.

The vertical structure of the unstable mode (Fig. 3, left panel) suggests that the instability is concentrated in the upper thermocline. The modal structure for the inviscid solution does not depend on the wave orientation. That this should be so follows from examination of (3). Since $\Pi_{x}=0$, a solution could have $\omega$ proportional to $k$, and then the reduced governing equation and boundary condition are both independent of orientation, although results still depend on $K$, hence the scalar wavelength. This scale dependence can be seen in Fig. 4 (green curve), where the most unstable (as a function of $\theta$ ) wavelength for this configuration is about $150 \mathrm{~km}$ (so that $K R \approx 0.8$, where $R$ is the first mode internal Rossby Radius of deformation $c_{1} / f$, computed using the $c_{1}$ from (6). Also, repeating the same calculation with $\beta=0$ (solid magenta line) leads to a more stable solution. Thus, $\beta$ exerts a destabilizing influence in this particular case, which is consistent with the two terms in (3c) having the same sign.

When a weak viscosity, $\nu=1 \times 10^{-4} \mathrm{~m}^{2} \mathrm{~s}^{-1}$, is included, the inviscid solutions are only slightly modified, with frequency corrections $O\left(1 \times 10^{-10} \mathrm{~s}^{-1}\right)$. However, two new solution types emerge. First, a narrow frequency range, densely packed with solutions, appears for all $\theta$. For these solutions (shown by a green band in Fig. 2), the magnitude of the real part of frequency is somewhat less than the real part of the inviscid unstable frequency. This band is associated with the multiple neighboring pressure structure curves in the middle panel of Fig. 3. These solutions have the real part of $\omega^{\prime}$ passing through zero, but they avoid being singularities because there is a small negative, $O\left(-1 \times 10^{-10} \mathrm{~s}^{-1}\right)$ imaginary part of $\omega$, corresponding to wave damping. In the absence of viscosity, these solutions would be rejected as singular. Note also, that the solutions now depend on orientation, as can be seen by the range of $\operatorname{Im}(P)$ in Fig. 3 (center and right panels). That this should be so can readily be seen from (9), where it is no longer possible to reduce the equation to a form independent of orientation. A more curious viscous solution is the discrete mode that forms where $\operatorname{Re}\left[\omega^{\prime}(z=0)\right] \approx 0$ (on the edge of the gray-shaded region where critical layers are possible). In the absence of viscosity, this somewhat surface intensified solution (Fig. 3, right panel) would represent a critical layer at the ocean surface. Although we do not believe this particular solution in detail (because of its incomplete, nondiffusive treatment of the critical-layer physics), we are nonetheless inclined to believe its general properties because of its continuity with valid solutions (e.g., case 4; Fig. 8).

A somewhat different picture occurs for strictly eastward flow under the same conditions (case 2: $U_{1}=0.05 \mathrm{~m} \mathrm{~s}^{-1}, U_{2}=V_{1}=V_{2}=0, s_{u 1}=0.0035 \mathrm{~m}^{-1}$, and $\lambda=200 \mathrm{~km}$; Fig. 5). Here, an inviscid stable mode with no zero crossings in the vertical (i.e., $n=0$ ) occurs, and the other mode is only very weakly unstable $\left[\operatorname{Im}(\omega)=O\left(1 \times 10^{-9} \mathrm{~s}^{-1}\right)\right]$. Again, the structures of inviscid solutions are independent of orientation, and the necessary condition for instability is met via the surface condition (as denoted by the horizontal cyan line). However, for all $\theta$, the integrand in (4) also changes sign, so that the necessary condition is also met this way, as denoted by the solid magenta line. Once viscosity is added, the weakly damped surface mode (green curve) again appears, but there is no dense band of viscous solutions of the sort found in case 1 . The unstable mode is slightly affected, and the stable mode becomes slightly unstable $\left[\operatorname{Im}(\omega)=O\left(4 \times 10^{-11} \mathrm{~s}^{-1}\right)\right]$ for all $\theta$. That damping 


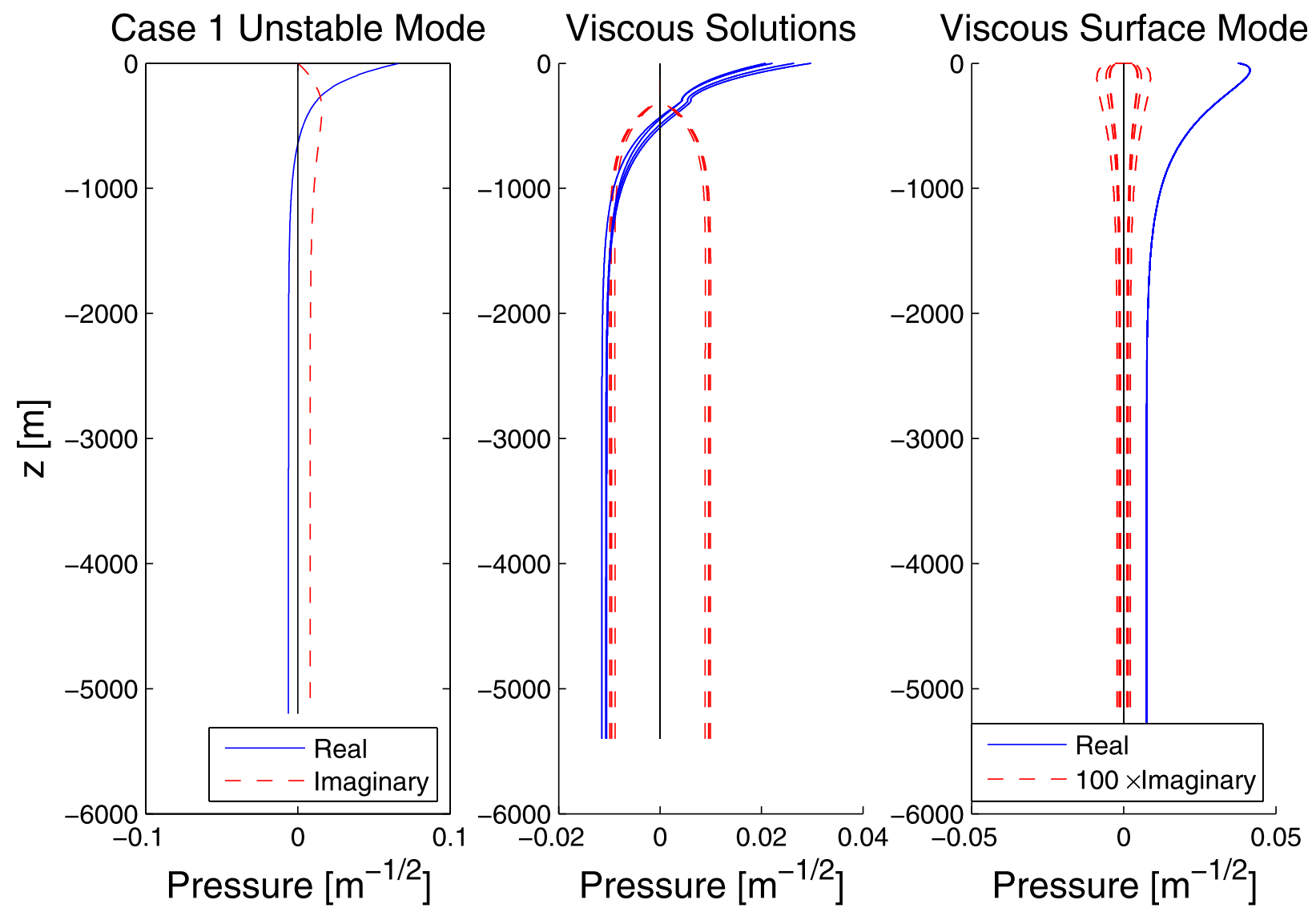

FIG. 3. Pressure modal structures for case 1: (left) the real part of pressure (blue) and imaginary part (red dashed) for the unstable mode (this structure is the same for all $\theta$ ), (center) the real part of pressure (blue) and imaginary part (red) for viscous solutions corresponding to the green band in Fig. 2, and (right) the real part of pressure (blue) and imaginary part (multiplied by 100) for the Fig. 2 viscous surface solution that occurs on the boundary between the gray-shaded and white areas. For the center and right panels, structures are presented for a sampling of $\theta$ values.

can enable baroclinic instability is, of course, a wellknown result (e.g., Holopainen 1961).

\section{2) MERIDIONAL MEAN FLOW}

Substantially more interesting results occur for strictly northward flow (case 3: Figs. 6 and 7), where $V_{1}=$ $0.05 \mathrm{~m} \mathrm{~s}^{-1}, U_{1}=U_{2}=V_{2}=0, s_{v 1}=0.0035 \mathrm{~m}^{-1}$, and $\lambda=200 \mathrm{~km}$ ). (Results for a similar configuration with southward flow are similar, but have a different phase with regard to $\theta$ ). There is now a mixture of stable and unstable solutions that vary considerably with the wavenumber orientation $\theta$. For $\theta=0^{\circ}$ and $180^{\circ}$, $l=0, \omega^{\prime}=\omega$, and there is an infinite set of stable inviscid vertical modes just as there would be with no mean flow. As $\theta$ increases toward $90^{\circ}$, the number of stable solutions decreases, and eventually an unstable mode appears in the form of a complex conjugate pair. For a substantial part of this mode's range, the instability criterion (4) is satisfied only by the sign contrast between the surface term and the integrand. The maximum growth rate occurs for $\lambda=125 \mathrm{~km}$ (Fig. 4).

Including viscosity allows two classes of new solutions, in addition to causing small changes to the inviscid solutions (e.g., the stable modes are now very weakly destabilized). First, there is a dense band of weakly damped complex solutions (with imaginary part of $\omega$ being $O\left(-2 \times 10^{-10} \mathrm{~s}^{-1}\right)$ : the wide green curve in Fig. 6 for $\theta=100^{\circ}-150^{\circ}$ ) that essentially completes the curve defined by the inviscid $n=0$ stable solution (solid blue curve). Following the $n=0$ curve as $\theta$ increases toward $90^{\circ}, \omega^{\prime}$ increases toward 0 and a critical layer is approached. Adding viscosity allows this $n=0$ curve to be completed via the dense band of solutions. In addition, as in the case with zonal mean flows, another, weakly damped, new mode is found that has $\operatorname{Re}\left(\omega^{\prime}\right) \approx 0$ at $z=0$.

The modal structures for $P$ (Fig. 7) vary considerably with $\theta$. Consider the inviscid $n=0$ mode (Fig. 7a) where for $\theta=0$, the mode is completely depth independent. 


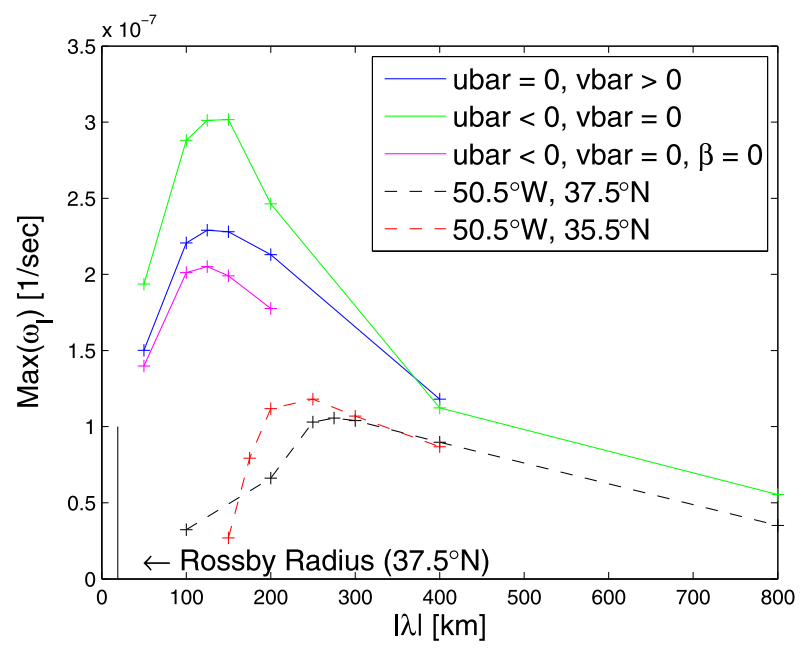

FIG. 4. Maximum (as a function of wavenumber direction $\theta$ at a given $\lambda$ ) growth rate vs scalar wavelength $\lambda$. Solid curves are for stratification and $f$ at $37.5^{\circ} \mathrm{N}, 50.5^{\circ} \mathrm{W}$ and idealized velocity profiles. The blue solid curve is case 3 , with $V_{1}=0.05 \mathrm{~m} \mathrm{~s}^{-1}, U_{1}=U_{2}=V_{2}=$ 0 , and $s_{v 1}=0.0035 \mathrm{~m}^{-1}$; the green solid curve is case 1 , with $U_{1}=-0.05 \mathrm{~m} \mathrm{~s}^{-1}, U_{2}=V_{1}=V_{2}=0$, and $s_{u 1}=0.0035 \mathrm{~m}^{-1}$; and the magenta solid curve is the same as the other two except that $\beta=0$. The dashed lines are for more realistic mean flows (black dashed as in case 4 and red dashed as in case 5). The first-mode Rossby radius scale at $37.5^{\circ} \mathrm{N}, 50.5^{\circ} \mathrm{W}$ (vertical black line) is given for comparison.

However, as $\theta$ increases, the mode shows increasing depth dependence, especially near the surface. As a measure of this distortion, the ratio $\eta$ of maximum $P$ to minimum $P$ is plotted in the lower panel. At $\theta=0, \eta=1$, but distortion grows $(\eta>20)$ as $\theta$ approaches $90^{\circ}$. Thus, the $n=0$ mode is only strictly barotropic when $l=0$. If anything, modal distortion is even more pronounced for the $n=1$ (first baroclinic) mode (Fig. 7b). In this case, the distortion is measured by $\gamma$, the ratio of surface $|P|$ to bottom $|P|$. (Note that $\eta$ is a measure of overall modal distortion used only for the $n=0$ mode, but that $\gamma$ is a measure of surface versus bottom amplitude for any mode. One could easily imagine situations with $\eta$ being large, but $\gamma=1)$. For $\theta=0$, the modal structure is again undistorted, and $\gamma=6.7$ as in a resting ocean. However, as $\theta$ varies, the modal structure becomes distorted, and $\gamma>70$ for $\theta=40^{\circ}$ (solid blue line in the lower panel). However, as $\theta$ approaches $85^{\circ}$, the mode has $\gamma<6.7$, the undisturbed value, and the maximum amplitude is at middepth. Thus, for the first baroclinic mode, mean flow usually, but not always, makes the structure more surface-intensified.

The inviscid unstable mode (Fig. 7c) is always surfaceintensified, especially for $\theta<80^{\circ}$, where $\gamma$ reaches 180 . The smallest $\gamma\left(6.5\right.$ at $\left.\theta=140^{\circ}\right)$ differs only slightly from the unperturbed value (6.7) for the $n=1$ mode. The complex mode with the surface near-critical layer

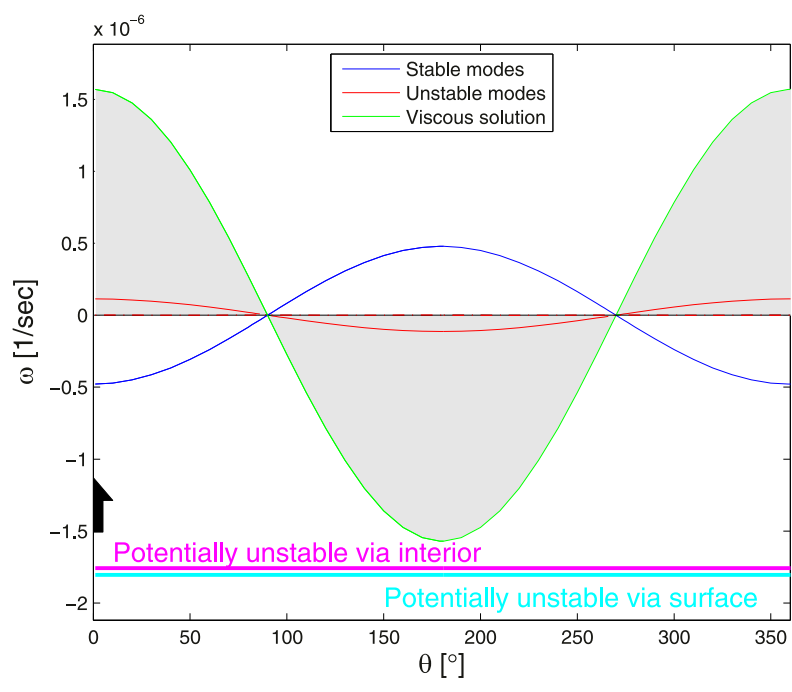

FIG. 5. Frequency of wave solutions vs wave direction for $\lambda=200 \mathrm{~km}$, case $2\left(37.5^{\circ} \mathrm{N}, 50.5^{\circ} \mathrm{W}, U_{1}=0.05 \mathrm{~m} \mathrm{~s}^{-1}, U_{2}=V_{1}=\right.$ $V_{2}=0$, and $\left.s_{u 1}=0.0035 \mathrm{~m}^{-1}\right)$. The solid red line is the real part of frequency for the inviscid complex mode; dashed red lines are the imaginary part of frequency for the same mode, where the positive values represent unstable waves and negative values represent decaying waves (barely distinguishable from zero); the solid blue curve represents a stable mode $(n=0)$; and the green line is the real part of frequency for the surface-trapped viscous solution [the imaginary part is negative and $\left.O\left(10^{-10} \mathrm{~s}^{-1}\right)\right]$. The gray-shaded area is the frequency range over which critical layers are possible, given the mean flow and $\theta$. When a viscosity of $\nu=1 \times 10^{-4} \mathrm{~m}^{2} \mathrm{~s}^{-1}$ is included, the red and blue curves are not noticeably changed at this scale. The horizontal cyan line indicates the $\theta$ range over which the system is potentially baroclinically unstable because the first term in (4) differs in sign from the integral, and the magenta line indicates the $\theta$ range that is potentially baroclinically unstable because the integrand in (4) changes sign. There are no inviscid critical layers for this case.

(Fig. 7d) is, to varying degrees, surface-intensified, with $\gamma$ in the range of $4-115$.

Thus, for this case, modal structures are nearly always distorted by the mean flow, and the mean flow usually enhances surface intensification for stable baroclinic modes $(n>0)$. Further, the unstable mode and the surface near-critical mode are also, for most $\theta$, more surface-intensified than the $n=1$ mode in a resting ocean.

\section{b. More realistic mean flows}

Consider first case $4\left(37.5^{\circ} \mathrm{N}, 50.5^{\circ} \mathrm{W}\right)$, where the mean surface flow is toward the southeast (Fig. 1 red curves, Fig. 8, Table 3), although the mean flow direction veers somewhat with depth because of the different vertical decay rates in (10b) and (10c). For most values of $\theta$, there are two unstable modes (solid red curves in Fig. 8). One mode [that with the larger magnitude of $\operatorname{Re}(\omega)$ ] exists in the $\theta$ range where the necessary condition (4) is 


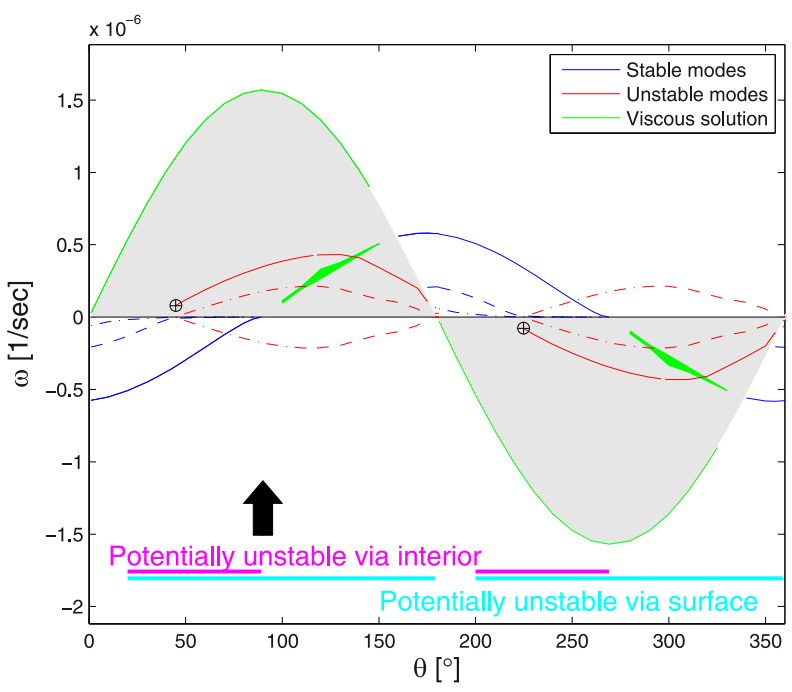

FIG. 6. Frequency of wave solutions vs wave direction for $\lambda=200 \mathrm{~km}$, case $3\left(37.5^{\circ} \mathrm{N}, 50.5^{\circ} \mathrm{W}, V_{1}=0.05 \mathrm{~m} \mathrm{~s}^{-1}, U_{1}=U_{2}=\right.$ $V_{2}=0$, and $\left.s_{v 1}=0.0035 \mathrm{~m}^{-1}\right)$. The solid red line is the real part of frequency for the inviscid complex mode; dashed red lines are the imaginary part of frequency for the same mode, where the positive values represent unstable waves and negative values represent decaying waves; blue curves represent stable modes (solid is $n=0$, dashed is $n=1$, and dash-dot is $n=2$; only the first three stable modes are shown); the thick green line is the real part of frequency for the dense band of solutions when viscosity is allowed; and the lighter-green line is the real part of frequency for the surfacetrapped viscous solution [for both viscous solutions, the imaginary part is negative and $\left.O\left(10^{-10} \mathrm{~s}^{-1}\right)\right]$. The black circle/cross symbols denote inviscid critical-layer solutions. The gray-shaded area is the frequency range over which critical layers are possible, given the mean flow and $\theta$. When a viscosity of $\nu=1 \times 10^{-4} \mathrm{~m}^{2} \mathrm{~s}^{-1}$ is included, the red and blue curves are not noticeably changed at this scale. The horizontal cyan line indicates the $\theta$ range over which the system is potentially baroclinically unstable because the first term in (4) differs in sign from the integral, and the magenta line indicates the $\theta$ range that is potentially baroclinically unstable because the integrand in (4) changes sign.

met because of the sign contrast between the surface term and the integral. Indeed, this mode's structure is very strongly trapped near the surface, within $200 \mathrm{~m}$ for most $\theta$, and for nearly all $\theta, \gamma>50 . \operatorname{Re}\left(\omega^{\prime}\right)$ passes through zero at some $z$ for all $\theta$ along the red (unstable) curve, but a critical layer does not occur because $\operatorname{Im}(\omega)$ is nonzero. However, as $\theta$ increases toward $66^{\circ}, \operatorname{Im}(\omega)$ approaches 0 , and so a critical layer (hence singular solutions) would occur in the absence of viscosity. When viscosity is included (green curve segment), a physically acceptable solution bridges the gap that would otherwise occur. A second unstable mode, having a smaller magnitude of $\operatorname{Re}(\omega)$, also occurs, and is associated with the integrand in the necessary condition (4) changing sign. This mode expresses baroclinic instability in the thermocline: its maximum vertical gradients occur over the upper $1000-2000 \mathrm{~m}$. In terms of $\operatorname{Im}(\omega)$, this mode is the more unstable of the two. Stable modes also exist, and they can occur in the frequency range where critical layers are possible (unlike the case in Fig. 6).

The $n=0$ stable mode for this case is always substantially distorted ( $\eta$ ranges from 1.3 to 3.3 ), with vertical variations distributed over primarily the upper $3000 \mathrm{~m}$. The $n=1$ stable mode is generally strongly distorted ( $\gamma$ in the range of 6.2-8800, and usually $>6.7$, the value in a resting ocean), with bottom flows being greatly weakened for $\theta=30^{\circ}$ to $50^{\circ}$, and relatively strong flows distributed over the upper $4000 \mathrm{~m}$ for $\theta$ in the range $140^{\circ}-190^{\circ}$. The most unstable mode has $\gamma$ in the range of 2.1-1100, although $\gamma<15$ for a wide range of $\theta: 70^{\circ}-$ $180^{\circ}$. All considered, surface intensification (as measured by $\gamma$ ) is greater than in a resting ocean for most directions of stable wave propagation.

For reference, the scalar wavelength of maximum instability is about $275 \mathrm{~km}$ at this location (Fig. 4), and $\beta$ acts to stabilize the flow. We repeated the above calculation for longer waves, $\lambda=400 \mathrm{~km}$, yielding qualitatively similar results, although at least two of the stable solutions exist over a much wider range of propagation angles $\left(120^{\circ}-240^{\circ}\right)$. Also, for most $\theta$, the unstable growth rate decreases relative to the case of $\lambda=200 \mathrm{~km}$. However, in the $\theta$ range of $80^{\circ}-110^{\circ}$, instability is enhanced. In addition, modal structures for $\lambda=400 \mathrm{~km}$ are somewhat less distorted relative to the case with no mean flow; for example, $\eta$ is as small as 1.1. Our calculations usually show that, for larger $\lambda$, both instabilities (e.g., Fig. 4) and modal distortions weaken.

A contrasting example (case $5: 35.5^{\circ} \mathrm{N}, 50.5^{\circ} \mathrm{W}$, and $\lambda=200 \mathrm{~km}$ ) has mean surface flow toward the southwest (Fig. 9, Table 3). In this case, there is a single inviscid unstable mode (for $\theta$ from $-5^{\circ}$ to $102^{\circ}$ ) that is only slightly modified when dissipation is included. The mode appears to be associated with shear in the thermocline, and is not very surface-intensified ( $\gamma$ of $1-4.2$ ). The direction of maximum instability is within about $10^{\circ}$ of the near-surface mean flow direction. In addition, there is a set of inviscid stable modes in the range of $100^{\circ}-160^{\circ}$. In particular, the $n=0$ mode is fairly depth independent ( $\eta$ is in the range of 1-2) for some $\theta$. But, for $\theta$ from $-5^{\circ}$ to $50^{\circ}$, the mode's character is very surface-intensified ( $\eta$ from 5 to 348), and the frequency falls just outside the range where critical layers are possible. This inviscid mode ceases to be a valid (continuous) solution for $\theta>50^{\circ}$, because $\omega^{\prime}$ reaches zero while remaining real (in contrast with case 4 , in which an inviscid curve ended because the imaginary part of $\omega$ reached zero) so that the solution would be singular. The higher-mode stable inviscid structures are all substantially modified relative to the resting-ocean case. For example, the $n=1$ mode 

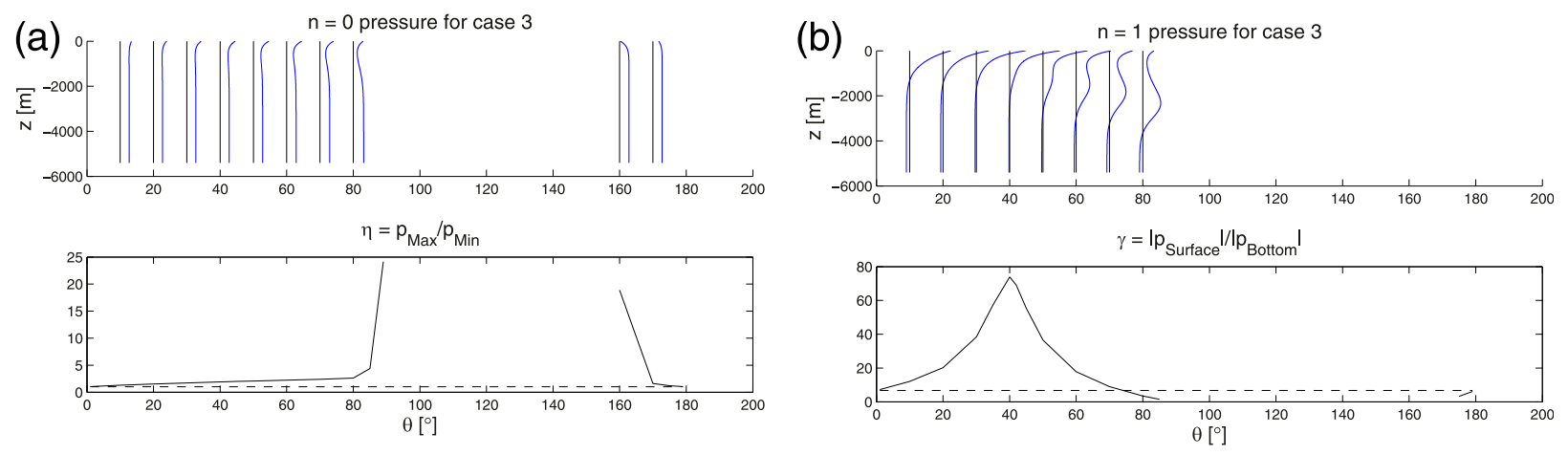

(c)
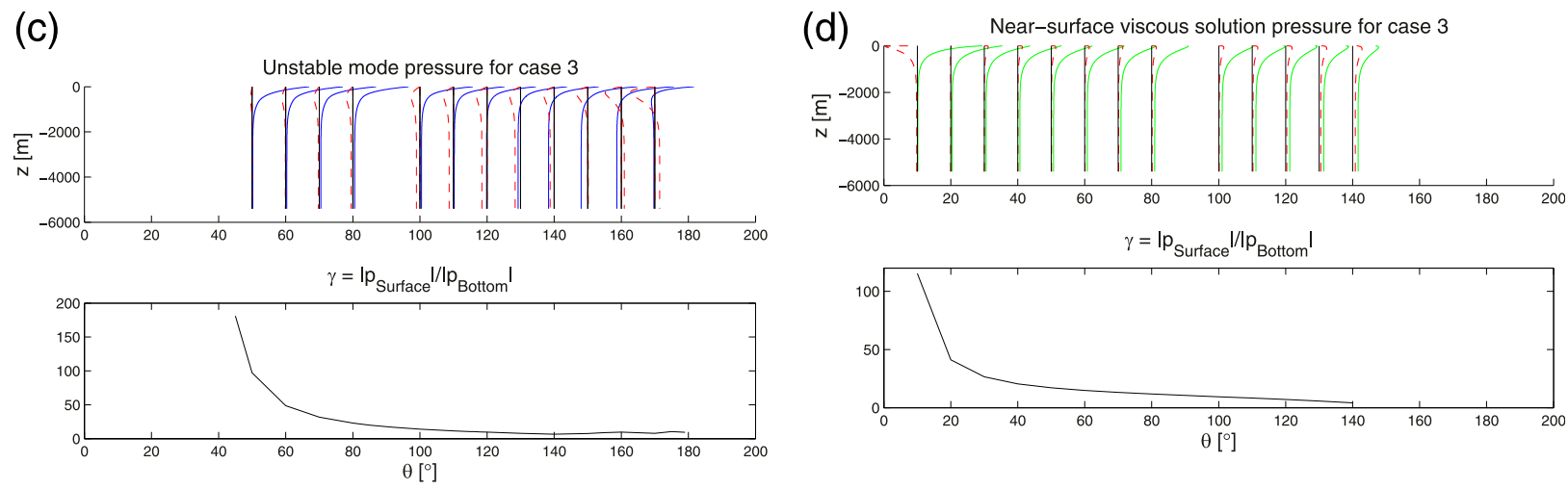

FIG. 7. Modal structures for case 3 , as a function of wavenumber orientation $\theta$ : (a) stable $n=0$ mode, (b) stable $n=1$ mode, (c) unstable mode, and (d) viscous mode with $\nu=1 \times 10^{-4} \mathrm{~m}^{2} \mathrm{~s}^{-1}$. For (a)-(d), the top plot shows a profile of pressure every $10^{\circ}$ where a solution of that type exists, with blue or green lines indicating the real part of pressure and red dashed curves indicating the imaginary part of the modal structure, and the bottom plot is a statistic representing the modal distortion. The horizontal dashed line in the lower plot of (a) and (b) is what that number would be in the absence of a mean flow. In contrast to Figs. 2,5 , and 6 , only $\theta=0^{\circ}-180^{\circ}$ is shown to allow the structures to be more visible.

has $\gamma$ in the range of $1.5-32$ as compared as $\gamma=6.8$ for a resting ocean.

Once dissipation is included, a tightly defined dense band of weakly damped modes complements the $n=0$ curve for $\theta=60^{\circ}-140^{\circ}$, while the inviscid part of the $n=0$ curve takes on a very slight, $O\left(10^{-12} \mathrm{~s}^{-1}\right)$, positive imaginary part, corresponding to a very weak instability. The higher-mode stable wave solutions become weakly damped. In addition, patchy dense bands of damped solutions appear near the real part of the unstable curve.

\section{c. Summary on modal structures}

The main result of these calculations is that the presence of a potential vorticity gradient, associated with a vertically varying mean flow, leads to significant distortion of the baroclinic modes even though the mean flows are relative weak: $O\left(0.05 \mathrm{~m} \mathrm{~s}^{-1}\right)$. We also studied results from other locations in the North Atlantic, but cases 4 and 5 are representative. The degree of distortion is sensitive to Rossby wave orientation relative to the mean flow direction, but the modifications are very often in the sense of enhanced surface intensification. In the process of investigating these mean flow effects, we inevitably encounter critical layers and baroclinic instabilities (which are themselves sensitive to the mean flow distribution), but our main concern is with the distortion of modal structures. Indeed, the instabilities we encounter (e.g., Fig. 4) are fairly slowly growing, for example, having a growth time scale of about 60 days or longer, but our simple model (which neglects lateral shears) is meant to treat modal structures and not to resolve the main causes of ocean eddy generation.

\section{Critical layers}

For some values of wavenumber, a critical layer, in which the mean advecting velocity matches the wave's phase speed, can occur. The governing equation in (3a) becomes singular unless the advection of mean potential vorticity also happens to vanish. This apparent coincidence is dynamically significant when it occurs because it often indicates the presence of a nearby (in $k$, $l$ space) unstable wave. The following analysis is a slight 


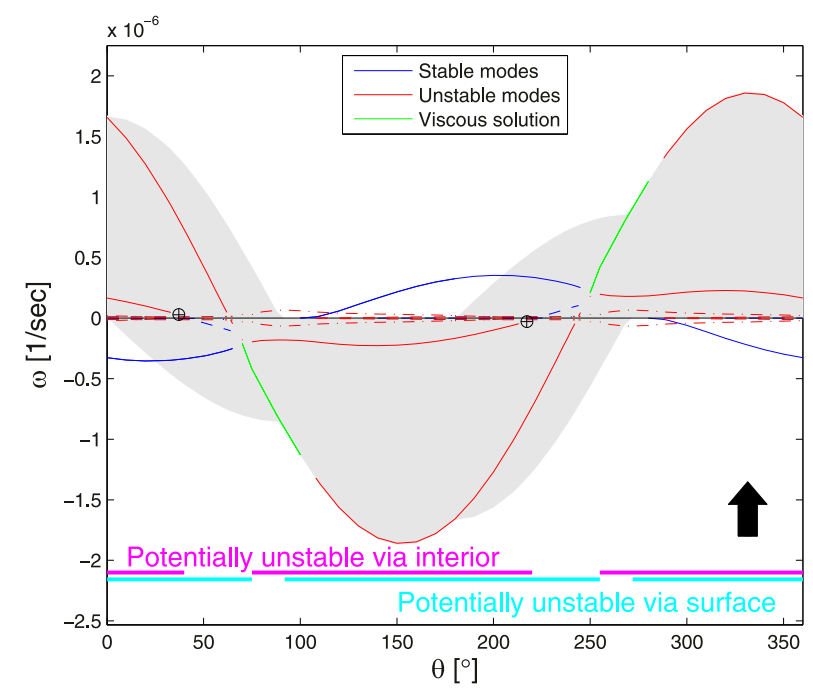

FIG. 8. Frequency of wave solutions vs wave direction for $\lambda=200 \mathrm{~km}$, case $4\left(37.5^{\circ} \mathrm{N}, 50.5^{\circ} \mathrm{W}\right.$, with velocity and $N^{2}$ set by a fit to observed climatology: Table 2). The solid red line is the real part of frequency for the inviscid complex mode; dashed red lines are the imaginary part of frequency for the same mode, where the positive values represent unstable waves and negative values represent decaying waves; blue curves present stable modes (solid is $n=0$; dashed is $n=1$ ), and the thick green line is the real part of frequency for the dense band of solutions when viscosity is allowed [the imaginary part is negative and $O\left(10^{-10} \mathrm{~s}^{-1}\right)$ ]. The black circle/cross symbols denote inviscid critical-layer solutions. The gray-shaded area is the frequency range over which critical layers are possible, given the mean flow and $\theta$. When a viscosity of $\nu=1 \times 10^{-4} \mathrm{~m}^{2} \mathrm{~s}^{-1}$ is included, the red and blue curves are not noticeably changed at this scale. The horizontal cyan line indicates the $\theta$ range over which the system is potentially baroclinically unstable because the first term in (4) differs in sign from the integral, and the magenta line indicates the $\theta$ range that is potentially baroclinically unstable because the integrand in (4) changes sign

generalization of the argument of Tollmien as described in the monograph of Lin (1955).

For the sake of conciseness we introduce the following variables. The projection of the mean velocity along the direction of the wave vector

$$
\begin{aligned}
\bar{U} & \equiv \bar{u} k / K+\bar{v} l / K \\
\operatorname{grad} Q & =\left(\frac{k}{K} \Pi_{y}-\frac{l}{K} \Pi_{x}\right),
\end{aligned}
$$

where the expression in (11b) is the cross product of the wave vector and the potential vorticity gradient of the mean field. This allows (3a) and (3e) to be compactly written as

$$
\begin{aligned}
\frac{\partial}{\partial z}\left(\frac{f^{2}}{N^{2}} \frac{\partial P}{\partial z}\right)-K^{2} P+P \frac{\operatorname{grad} Q}{\bar{U}-c} & =0 \quad \text { and } \\
(\bar{U}-c) P_{z}-\overline{U_{z}} P & =0, \quad z=-D, 0,
\end{aligned}
$$

with $c=\omega / K$. It follows from (12a) and (12b) that

$$
\begin{gathered}
K^{2}=\frac{\int_{-D}^{0} M P^{2} d z-\int_{-D}^{0} \frac{f^{2}}{N^{2}} P_{z}^{2} d z+\left.\frac{f^{2}}{N^{2}} \frac{\bar{U}}{\bar{U}-c} P^{2}\right|_{z=-D} ^{z=0}}{\int_{-D}^{0} P^{2} d z} \\
M(z, k, l)=\frac{\operatorname{grad} Q}{\bar{U}-c}
\end{gathered}
$$

which places an obvious condition on $M$ and the ratio $\bar{U}_{z} /(\bar{U}-c)$ at the upper and lower boundaries: 1) suppose there is an orientation of the wave vector $\mathbf{K}$ such that $\operatorname{grad} Q$ vanishes for some $z=z_{0}$ in $(-D, 0)$, and 2) let $c=\bar{U}\left(z_{o}\right)$. Then $M$ is a nonsingular function and solutions to (12a) and (12b), if they exist with real $K^{2}$, will be nonsingular. Let that solution be denoted as $P_{s}$ corresponding to $K^{2}=K_{s}^{2}$ and $c=c_{s}=\bar{U}\left(z_{o}\right)$ so that

$$
\begin{aligned}
\frac{\partial}{\partial z}\left(\frac{f^{2}}{N^{2}} \frac{\partial P_{s}}{\partial z}\right)-K_{s}^{2} P_{s}+M_{s} P_{s} & =0 \\
M_{s} & =\operatorname{grad} Q /\left(\bar{U}-c_{s}\right) .
\end{aligned}
$$

If we multiply (14a) and (14b) by $P$ and (12a) and (12b) by $P_{s}$ and integrate the result over the interval $(-D, 0)$, we obtain

$$
\begin{aligned}
\left(K^{2}-K_{s}^{2}\right) \int_{-D}^{0} P P_{s} d z= & \int_{-D}^{0} d z P P_{s}\left(M-M_{s}\right) \\
& -\left[\frac{f^{2}}{N^{2}} P P_{s}\left(\frac{\bar{U}_{z}}{\bar{U}-c}-\frac{\bar{U}_{z}}{\bar{U}-c_{s}}\right)\right]_{-D}^{0}
\end{aligned}
$$

In the limit as $K^{2} \rightarrow K_{s}^{2}+d K^{2}, c \rightarrow c_{s}+d c$, (where $d K^{2}$ and $d c$ represent infinitesimal perturbations), (15) becomes

TABLE 3. Parameters for cases 4 and 5.

\begin{tabular}{ccccccccccc}
\hline \hline Case & $U_{1}\left(\mathrm{~m} \mathrm{~s}^{-1}\right)$ & $U_{2}\left(\mathrm{~m} \mathrm{~s}^{-1}\right)$ & $V_{1}\left(\mathrm{~m} \mathrm{~s}^{-1}\right)$ & $V_{2}\left(\mathrm{~m} \mathrm{~s}^{-1}\right)$ & $N_{0}^{2}\left(\mathrm{~s}^{-2}\right)$ & $s_{u 1}\left(\mathrm{~m}^{-1}\right)$ & $s_{u 2}\left(\mathrm{~m}^{-1}\right)$ & $s_{v 1}\left(\mathrm{~m}^{-1}\right)$ & $s_{v 2}\left(\mathrm{~m}^{-1}\right)$ & $s_{N}\left(\mathrm{~m}^{-1}\right)$ \\
\hline 4 & -0.3658 & 0.4162 & 0.3317 & -0.3591 & $3.66 \times 10^{-5}$ & 0.0040 & 0.0033 & 0.0033 & 0.0031 & 0.0021 \\
5 & 0.1839 & -0.2065 & 0.1554 & -0.1804 & $3.55 \times 10^{-5}$ & 0.0029 & 0.0025 & 0.0033 & 0.0029 & 0.0022 \\
\hline
\end{tabular}




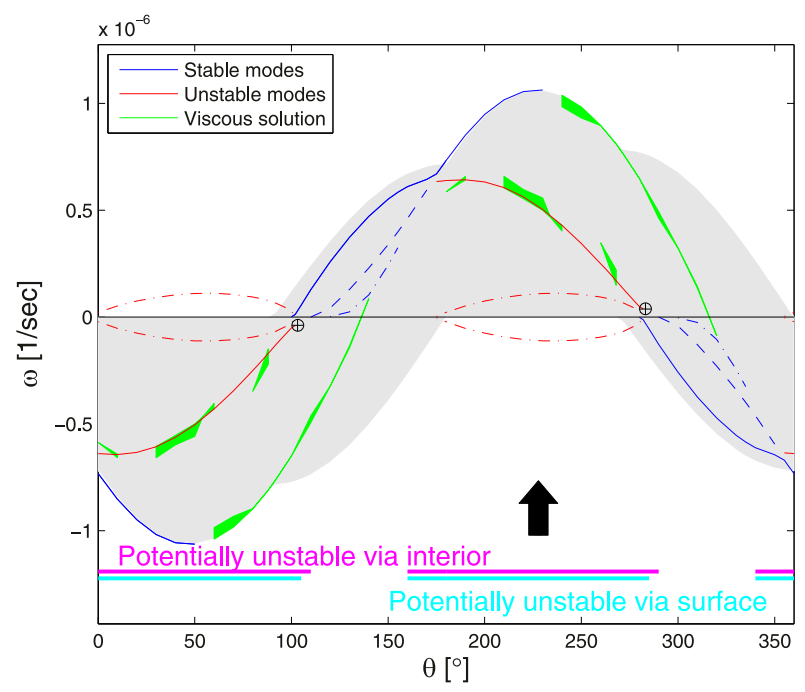

FIG. 9. Frequency of wave solutions vs wave direction for $\lambda=200 \mathrm{~km}$, case $5\left(35.5^{\circ} \mathrm{N}, 50.5^{\circ} \mathrm{W}\right.$, with velocity and $N^{2}$ set by a fit to observed climatology: Table 2). The solid red line is the real part of frequency for the inviscid complex mode; dashed red lines are the imaginary part of frequency for the same mode, where the positive values represent unstable waves and negative values represent decaying waves; blue curves present stable modes (solid is $n=0$; dashed is $n=1$, dash-dot is $n=2$ ); and the thick green line is the real part of frequency for the dense band of solutions when viscosity is allowed [the imaginary part is negative and $\left.O\left(10^{-10} \mathrm{~s}^{-1}\right)\right]$. The black circle/cross symbols denote inviscid critical-layer solutions. The gray-shaded area is the frequency range over which critical layers are possible, given the mean flow and $\theta$. When a viscosity of $\nu=1 \times 10^{-4} \mathrm{~m}^{2} \mathrm{~s}^{-1}$ is included, the red and blue curves are not noticeably changed at this scale. The horizontal cyan line indicates the $\theta$ range over which the system is potentially baroclinically unstable because the first term in (4) differs in sign from the integral, and the magenta line indicates the $\theta$ range that is potentially baroclinically unstable because the integrand in (4) changes sign.

$$
\begin{aligned}
d K^{2} \int_{-D}^{0} P_{s}^{2} d z= & d c\left(\int_{-D}^{0} \frac{P_{s}^{2} M_{s}}{\bar{U}-c} d z\right) \\
& +d c\left[\frac{f^{2}}{N^{2}} P_{s}^{2} \frac{\overline{U_{z}}}{\left(\bar{U}-c_{s}\right)^{2}}\right]_{-D}^{0} .
\end{aligned}
$$

If the phase speed of the mode adjacent to the neutral mode $P_{s}$ with a small change in wavenumber has a small imaginary part $c_{i}$, the first integral on the right hand side of (16) has an imaginary part given by the contribution of the residue in the neighborhood where $\bar{U}-c_{s}-c_{i}=0$ yielding (as $c_{i} \rightarrow 0$ )

$$
\operatorname{Im}\left\{\int_{-D}^{0} \frac{P_{s}^{2} M_{s}}{\bar{U}-c} d z\right\}=i \pi \frac{P_{s}^{2}\left(z_{o}\right) M_{s}\left(z_{o}\right)}{\bar{U}_{z}\left(z_{o}\right)}
$$

The remaining terms on the right hand side of (16) come from the real part of the integral, that is, the principal part of the integral plus the boundary terms [the last terms in (16)], which are strictly real in the limit of $c \rightarrow c_{s}$.

This allows us to write

$$
\begin{aligned}
\frac{d K^{2}}{d c} & =(A+i B) / \int_{-D}^{0} P_{s}^{2} d z \\
A & =\operatorname{Re} \subset \int_{-D}^{0} \frac{P_{s}^{2}}{\bar{U}-c_{s}} M_{s}(z) d z+\left.\frac{f^{2}}{N^{2}} \frac{P_{s}^{2} \bar{U}}{\left(\bar{U}-c_{s}\right)^{2}}\right|_{z=-D} ^{z=0} \\
B & =\frac{\pi P_{s}^{2}\left(z_{o}\right) M_{s}\left(z_{o}\right)}{\bar{U}_{z}\left(z_{o}\right)}
\end{aligned}
$$

where $\subset$ denotes the principal value of the integral. It follows that

$$
\frac{d c}{d K^{2}}=\frac{A-i B}{A^{2}+B^{2}}
$$

so that an increase in wavenumber will lead to a decrease or increase in the growth rate if $B$ is positive or negative, respectively, so that an unstable wave should be found contiguous to the neutral wave at the critical level as long as $B$ differs from zero.

This result is demonstrated for case 3 (Fig. 10). The problem (12) is discretized onto a vertical 8000-point finite-difference grid so that $K^{2}$ is the algebraic eigenvalue of the problem. Depending on $\theta$, there is at most one solution having $K^{2}>0$ for case 3 (Fig. 10a), but, in other cases not shown here, there are angles for which more than one eigenvalue corresponds to $K^{2}>0$. There are some situations (e.g., cases 4 and 5) in which $\operatorname{grad} Q$ can change sign twice as a function of depth. In these cases, it is possible for there to be a critical layer at either of two depths. This does not add any undue complications as long as there is only one zero of $(\bar{U}-c)$. The critical-layer frequencies for cases where $K=2 \pi /(200 \mathrm{~km})$ are plotted as black symbols in Figs. 6, 8 , and 9 (the imaginary parts are zero, of course). For cases 1 and 2, there are no inviscid critical-layer solutions with $K=2 \pi /(200 \mathrm{~km})$. In all cases, the criticallayer solution occurs in the $(\theta, \omega)$ plane at the "end" of the curve for $\operatorname{Re}(\omega)$ for an unstable mode, that is, at the $\theta$ value where an inviscid unstable mode exists to one side, but no contiguous continuous inviscid solution (stable or not) exists on the other.

The meaning of (19) is thus illustrated. Picking $\theta$ as the angle at which the inviscid critical layer exists, one can compute the complex frequency for values of $K$ slightly below and above $K_{s}$. For $d \operatorname{Im}(c) / d K^{2}>0$, $\operatorname{Im}(c)$ is nonzero, (corresponding to unstable solutions) for $K>K_{s}$. For $K<K_{s}$, the solutions all have $\operatorname{Im}(c)=0$, 

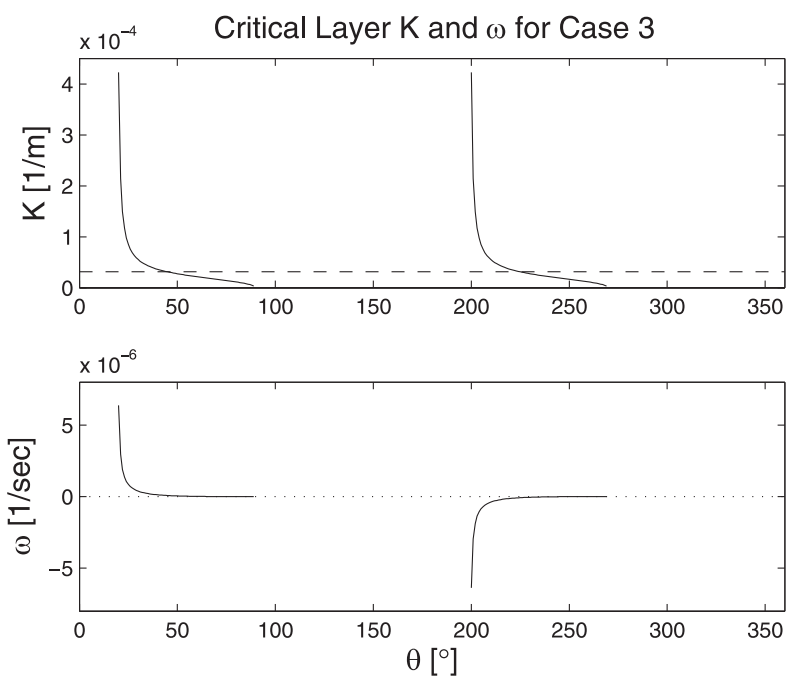

FIG. 10. Results for the inviscid critical layer for case 3 (see also Fig. 6) (top) scalar wavenumber $K$ as a function of the wavenumber angle $\theta$ [the dashed line is for $K=3.14159 \times 10^{-5} \mathrm{~m}^{-1}$ $(\lambda=200 \mathrm{~km})$ so that the intersections with the solid line indicate the solution denoted by black symbols in Fig. 6] and (bottom) wave frequency for critical-layer solutions.

but $\omega^{\prime}$ changes sign with depth, so the inviscid solution is invalid. That is, there is an unstable solution for larger $K$ but no inviscid solution for smaller $K$. Similarly, when $d \operatorname{Im}(c) / d K^{2}<0$, the nearby solutions for $K>K_{s}$ are all invalid and those for $K<K_{s}$ are unstable. This outcome is clearly consistent with the inviscid critical layers occurring where an unstable solution begins to occur.

Note that critical-layer solutions are only possible where $\operatorname{grad} Q$ changes sign (so that $M$ can be continuous) somewhere in the depth range $-D<z<0$. Comparing this to the necessary condition for instability (4), we see that inviscid critical-layer solutions can only occur where the necessary condition is met by $\operatorname{grad} Q$ changing sign (the range labeled "potentially unstable via interior" in Figs. 6, 8, and 9). For this reason, there are situations (e.g., Fig. 6 near $\theta=175^{\circ}$ ) where an unstable curve ends but no inviscid critical layer exists. Thus, the existence of an inviscid critical-layer solution implies a nearby unstable mode, but an unstable mode does not require that there is an inviscid critical-layer solution.

\section{The $n=0$ mode}

Brink (1989, hereinafter B89) used both observations and theory to study the linear response of subinertial ocean currents below the mixed layer to wind stress curl forcing in the North Atlantic. He found that currents and temperatures are often coherent with the wind stress curl at distant (more than $1000 \mathrm{~km}$ in many cases) locations. All of B89's observational results are for periods of 49 days or shorter. The apparent underlying physics is rather straightforward: Rossby waves of many scales are excited by the wind stress curl all over the ocean, and the long waves propagate information westward, while shorter waves carry information eastward. Thus, pressure and east-west currents (proportional to $l$ ) at a given location are more sensitive to long waves (where $k \ll l)$ arriving from the east, but north-south currents (proportional to $k$ ) are more affected by shorter waves $(l \ll k)$ arriving from the west.

What is curious about the B89 observations is that temperature signals well below the mixed layer (e.g., below 150-m depth) are coherent with distant wind stress curl forcing, even at frequencies far too high [as compared with $\beta c_{1}(2 f)^{-1} \approx O\left(2 \times 10^{-7} \mathrm{~s}^{-1}\right)$, or a period of about a year] to allow classical baroclinic Rossby wave propagation. While barotropic Rossby waves can certainly propagate at a 49-day period, strictly barotropic waves carry no density (hence temperature) signal. No significant coherence of temperature with local wind stress curl was found. Our present findings appear to provide a potential resolution to this conundrum.

Consider how the $n=0$ mode is distorted by the presence of a baroclinic mean flow. The induced vertical gradients in pressure correspond to density (hence temperature) variations through the hydrostatic relation

$$
0=p_{z}+g \rho .
$$

With this in mind, we examine solutions for a 49-day period at the approximate latitude $\left(26.5^{\circ} \mathrm{N}\right)$ of the $\mathrm{B} 89$ observations. The results do not vary substantially with longitude in the western North Atlantic. We find, for example, that $(k, l)=(3.5,6) \times 10^{-6} \mathrm{~m}^{-1}$ gives a Rossby wave frequency of $1.48 \times 10^{-6} \mathrm{~s}^{-1}$ (49 day period), and the somewhat distorted $(\eta=1.07)$ pressure modal structure shown in Fig. 11. At this frequency, no highermode, $n>0$, Rossby waves are possible. At a depth of about $500 \mathrm{~m}$, this structure yields $P_{z} / P \approx 2.7 \times 10^{-3} \mathrm{~m}^{-1}$. This can be used to estimate a $T / v$ amplitude ratio of $0.5^{\circ} \mathrm{C} \mathrm{s} \mathrm{m}^{-1}$ and a $T / u$ ratio of about $0.3^{\circ} \mathrm{C} \mathrm{s} \mathrm{m}^{-1}$. These ratios are also estimated spectrally using current-meter data from around 500-m depth and a period of 49 days from both of the B89 moorings. At 95\% confidence, this observed ratio for a 49-day period is in the range of $0.43-0.64$ for both $u$ and $v$. Some caveats are necessary because comparing this number with the theory makes several serious assumptions, including 1) that all of the ocean signal at this frequency is associated with a single $(k, l)$ pair and 2) that all of the signal in the data represents linear, wind-driven Rossby waves. Although we find the comparison of amplitude ratios encouraging, a good deal of caution is thus required. 


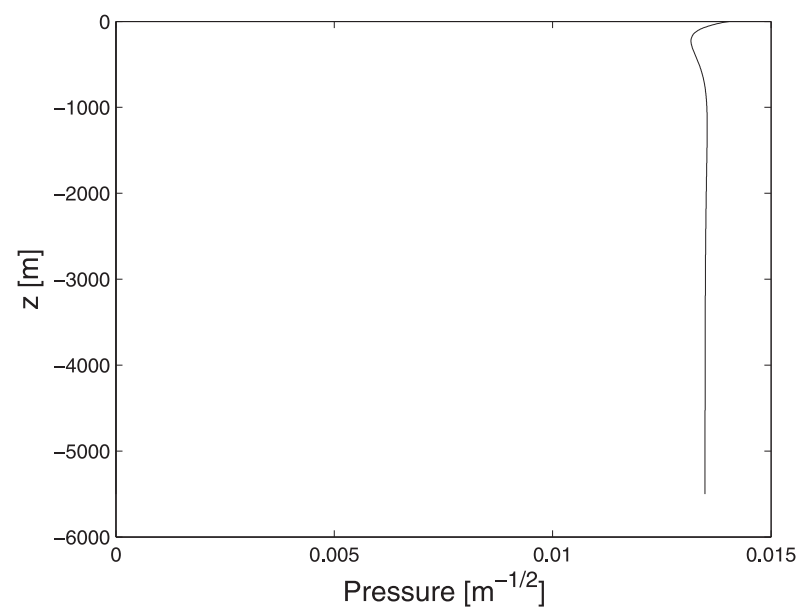

FIG. 11. Pressure modal function for the $n=0$ mode at $26.5^{\circ} \mathrm{N}$, $60.5^{\circ} \mathrm{W}$ with $(k, l)=(3.5,6) \times 10^{-6} \mathrm{~m}^{-1}$.

There is an alternative possibility that bears consideration. One might use the BPD assumption that the ocean's true baroclinic modal structures are governed by a bottom boundary condition of no horizontal flow (rather than the usual assumption of no vertical flow at the bottom). In this case, the gravest mode has amplitude decreasing monotonically toward the bottom. This gravest modal structure yields a $P_{z} / P$ ratio at $500-\mathrm{m}$ depth similar to that for Fig. 11. For the North Atlantic at $26.5^{\circ} \mathrm{N}$, this mode has an internal gravity wave speed of $c=3.92 \mathrm{~m} \mathrm{~s}^{-1}$, which translates into a minimum Rossby wave period of 115 days. Thus, Rossby waves cannot exist at a 49-day period under this bottom boundary condition assumption. Since the B89 observations show a clear westward propagation and thus behave qualitatively according to a wave-based theory, we conclude that the BPD bottom boundary condition cannot account for the observed temperature propagation at a period too short for a strictly linear baroclinic Rossby wave.

One might argue, as in LaCasce (2017), that the BPD condition applies because of a large-scale sloping bottom in a resting ocean. In this case, there will be an additional topographic Rossby wave mode that has its largest amplitude near the bottom. We explore this possibility by using a bottom slope, $\left(\alpha^{x}, \alpha^{y}\right)=$ $(1.4,1.25) \times 10^{-3}$, representative of the ocean southeast of Bermuda. Using a sampling of wavenumber pairs that allow $\omega=1.48 \times 10^{-6} \mathrm{~s}^{-1}$, we find that the topographic wave mode is almost barotropic so that the ratio $P_{z} / P$ at 500-m depth is consistently an order of magnitude smaller than we find with an ambient mean flow.

Very likely, there remain other possible explanations for how a disturbance propagating at a 49-day period can have a signature in temperature, but for now, the mean flow effect appears to be a possibility.

\section{Conclusions}

The primary finding of this study is that accounting for the effects of a realistic baroclinic oceanic mean flow leads to very substantial changes in the structure of the system's stable baroclinic modes relative to results in a resting ocean. Modal structures can change markedly (e.g., Fig. 7b) as a function of propagation direction, and often in the sense of making the modes more surfaceintensified than in the absence of a mean flow. Further, there are often instances of baroclinically unstable modes, and these, too can sometimes be extremely surfaceintensified (Fig. 7c). Thus, it seems possible that the ambient potential vorticity field associated with a mean flow can be relevant to the observed tendency for measured current variability to be more surface-intensified than would be the case for an $n=1$ baroclinic mode in a resting ocean. However, caution is required because of the model's dependence on wave orientation. For this reason, we do not consider the mean flow effect to be the last word on surface intensification. Rather, other possibilities certainly exist, most notably the reality that the oceanic eddy field is itself somewhat nonlinear (e.g., Chelton et al. 2011), and that the nonlinearity of individual eddies (e.g., McWilliams and Flierl 1979) could be the ultimate cause of the commonly observed surface intensification.

When potential vorticity advection [as represented by the $\operatorname{grad} Q$ term in (12)] passes through zero at some depth $z_{0}$, it is possible to have a well-behaved critical layer at that depth in the absence of any viscosity. We then show that this solution needs to adjoin an unstable solution. Indeed, these adiabatic critical layers, when they occur, appear at points in the $(\theta, \omega)$ plane when the growth rate of an unstable mode reaches zero.

One aspect of the mean flow effect is that the $n=0$ stable mode is no longer barotropic: its structure now varies in the vertical, and thus carries a signal in temperature as well as velocity and pressure. This ability to propagate temperature information at frequencies that are not possible for higher-mode Rossby waves appears to be a possible explanation for current-meter measurements from the North Atlantic thermocline that show temperature coherence with remote wind stress curl at a period of 49 days. Specifically, neither BPD modes nor topographic Rossby wave modes can account for the implied propagation. There are, however, some serious assumptions made in this argument, so a more careful analysis is called for, perhaps in the form of a three-dimensional numerical model linearized about mean oceanic conditions. 
Once a baroclinic mean flow is included in this problem, the vertical modes are no longer orthogonal as they are in the case of a resting ocean $[(6 c)]$. This makes it harder to use the resulting modes for oceanographic applications such as finding the response to wind forcing. However, there are certainly situations where our modified modal structures are still likely to appear in a natural way (as long as the horizontal scales of the mean flow are large enough for its lateral shear to be negligible). For example, one would expect the modes to be relevant for initial value or boundary value problems, where energy initially in a particular mode might well remain there. Further, the modified baroclinic modes are expected to be relevant in scattering problems (e.g., off of bottom relief). Thus, we anticipate that, at least in some contexts, modes of the sort we explore here should be observable in nature.

Last, we repeat a well-known caution. Baroclinic modes in the ocean take a finite time-determined by the vertical propagation time for a Rossby wave to transit the depth of the ocean-to set up. This is true whether or not there is a mean flow. Thus, if the background (mean) flow changes rapidly in time, using our modes will be problematic.

Acknowledgments. This research was partially supported by the National Science Foundation Physical Oceanography Program through Grant OCE-1433953. We appreciate helpful comments from Roger Samelson, the reviewers and the editor.

\section{REFERENCES}

Bobrovich, A. V., and G. M. Reznik, 1999: Planetary waves in a stratified ocean of variable depth. Part 2. Continuously stratified ocean. J. Fluid Mech., 388, 147-169, https://doi.org/ 10.1017/S0022112099004863.

Brink, K. H., 1989: Evidence for wind-driven current fluctuations in the western North Atlantic. J. Geophys. Res., 94, 2029-2044, https://doi.org/10.1029/JC094iC02p02029.

- and J. Pedlosky, 2018: Rossby waves with continuous stratification and bottom friction. J. Phys. Oceanogr., 48, 22092219, https://doi.org/10.1175/JPO-D-18-0070.1.
Charney, J. G., 1947: The dynamics of long waves in a baroclinic westerly current. J. Meteor., 4, 135-162, https://doi.org/ 10.1175/1520-0469(1947)004<0136:TDOLWI >2.0.CO;2.

_ , and P. G. Drazin, 1961: Propagation of planetary-scale disturbances from the lower into the upper atmosphere. J. Geophys. Res., 66, 83-109, https://doi.org/10.1029/JZ066i001p00083.

Chelton, D. B., M. G. Schlax, and R. M. Samelson, 2011: Global observations of nonlinear mesoscale eddies. Prog. Oceanogr., 91, 167-216, https://doi.org/10.1016/j.pocean.2011.01.002.

Held, I. M., 1985: Pseudomomentum and the orthogonality of modes in shear flows. J. Atmos. Sci., 42, 2280-2288, https://doi.org/10.1175/1520-0469(1985)042<2280:PATOOM> 2.0.CO;2.

Holopainen, E. O., 1961: On the effect of friction in baroclinic waves. Tellus, 13, 363-367, https://doi.org/10.1111/j.21533490.1961.tb00097.x.

Killworth, P. D., D. B. Chelton, and R. A. De Szoeke, 1997: The speed of observed and theoretical long extratropical planetary waves. J. Phys. Oceanogr., 27, 1946-1966, https://doi.org/ 10.1175/1520-0485(1997)027<1946:TSOOAT > 2.0.CO;2.

LaCasce, J. H., 2017: The prevalence of oceanic surface modes. Geophys. Res. Lett., 44, 11 097-11 105, https://doi.org/10.1002/ 2017GL075430.

Lin, C. C., 1955. The Theory of Hydrodynamic Stability. Cambridge University Press, $155 \mathrm{pp}$.

McWilliams, J. C., and G. R. Flierl, 1979: On the evolution of isolated, nonlinear vortices. J. Phys. Oceanogr., 9, 1155-1182, https://doi.org/10.1175/1520-0485(1979)009<1155:OTEOIN> 2.0.CO;2.

Pedlosky, J., 1992: Geophysical Fluid Dynamics. Springer-Verlag, 728 pp.

Samelson, R. M., 1992: Surface-intensified Rossby waves over rough topography. J. Mar. Res., 50, 367-384, https://doi.org/ 10.1357/002224092784797593.

Sanchez de la Lama, M., J. H. LaCasce, and H. K. Fuhr, 2016: The vertical structure of ocean eddies. Dyn. Stat. Climate Syst., 1, 1-16, https://doi.org/10.1093/climsys/dzw001.

Smith, K. S., 2007: The geography of linear baroclinic instability in Earth's oceans. J. Mar. Res., 65, 655-683, https://doi.org/ 10.1357/002224007783649484.

Wortham, C., and C. Wunsch, 2014: A multidimensional spectral description of ocean variability. J. Phys. Oceanogr., 44, 944966, https://doi.org/10.1175/JPO-D-13-0113.1.

Wunsch, C., 1997: The vertical partition of oceanic horizontal kinetic energy. J. Phys. Oceanogr., 27 1770-1794, https://doi.org/ 10.1175/1520-0485(1997)027<1770:TVPOOH>2.0.CO;2.

Zweng, M. M., and Coauthors, 2013: Salinity. Vol. 2, World Ocean Atlas 2013, NOAA Atlas NESDIS 74, 39 pp., http:// data.nodc.noaa.gov/woa/WOA13/DOC/woa13_vol2.pdf. 